\title{
A refined approach exploiting tubes of flux for analysis of linear switched reluctance motors
}

\author{
A. Stuikys*, M. Rotaru and J.K. Sykulski \\ ECS, University of Southampton, Southampton, UK
}

\begin{abstract}
A field modelling approach is presented exploiting approximate magnetic flux distributions. The method is an extension of flux-tubes and tubes-and-slices techniques. A combination of an equivalent magnetic circuit approach with improved description of relevant flux paths allows for a computationally efficient algorithm suitable for design optimization. The method is illustrated using an example of a linear switched reluctance motor and validated using finite element simulations.
\end{abstract}

Keywords: Switched reluctance motor, design optimization, flux tube modelling, flux linkage estimation, non-linear magnetic circuits, magnetic equivalent circuits

\section{Introduction}

A linear switched reluctance motor (LSRM) has applications in magnetic guidance and levitation systems for transportation and automation systems, rod actuator control mechanisms for nuclear reactors and other types of high force density linear actuators in hostile environments. The LSRM can be designed using similar equations as for a radial flux SR motor [1]. Due to the importance of pole tip saturation the modelling requires methods that can accurately capture and quantify such saturation effects [2]. The approach taken in this paper builds on an old flux-tubes modelling technique [3], used successfully to analyse tractive electromagnets and SR machines [1-5]. In this paper flux-tube approximations based on cubic-splines are introduced to improve the accuracy of the flux-tubes approach. The method is further extended by incorporating some concepts of the 'tubes and slices' (TAS) approach published in [6,7]. The proposed model overcomes some restrictions reported in [8] regarding distribution of flux-tubes (also known as equiflux or isoflux paths) in the air-gap region of the LSRM. As always with such techniques, prior knowledge and experience of the designer are beneficial.

\section{Modelling approach}

The model exploits the symmetries (Fig. 1) where the shaded magnetic flux plot has been obtained using standard finite element analysis (FEA). In the selected symmetry segment the cubic-spline representation has been applied to match the actual flux tubes distribution with the aid of careful positioning

\footnotetext{
${ }^{*}$ Corresponding author: A. Stuikys, ECS, University of Southampton, Southampton SO17 1BJ, UK. E-mail: A.Stuikys@ soton.ac.uk.
} 


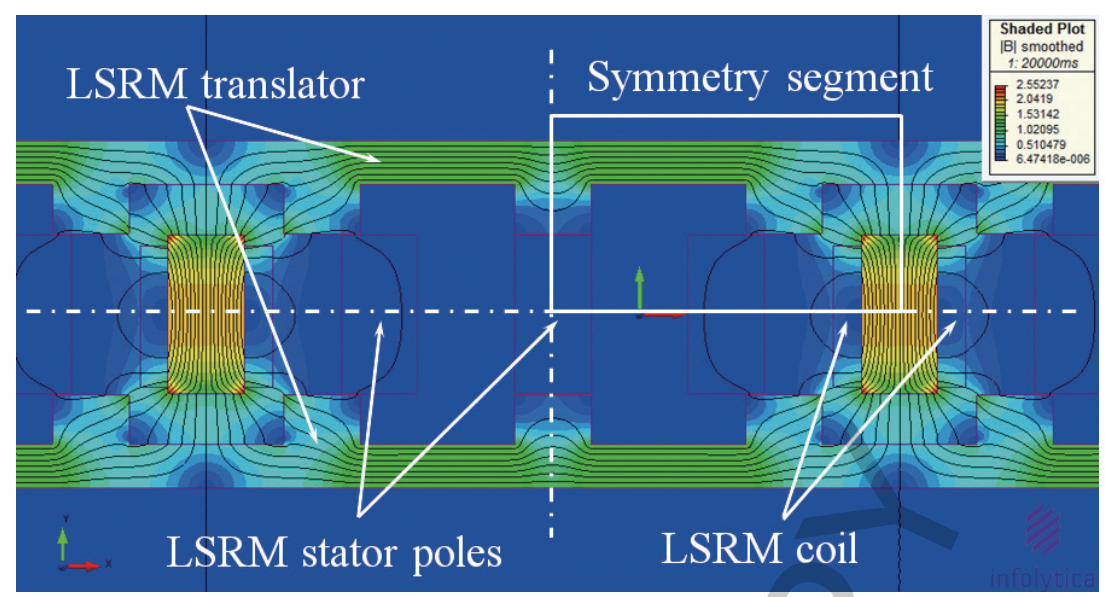

Fig. 1. Magnetic flux plot for a symmetry segment of the LSRM for the unaligned position.

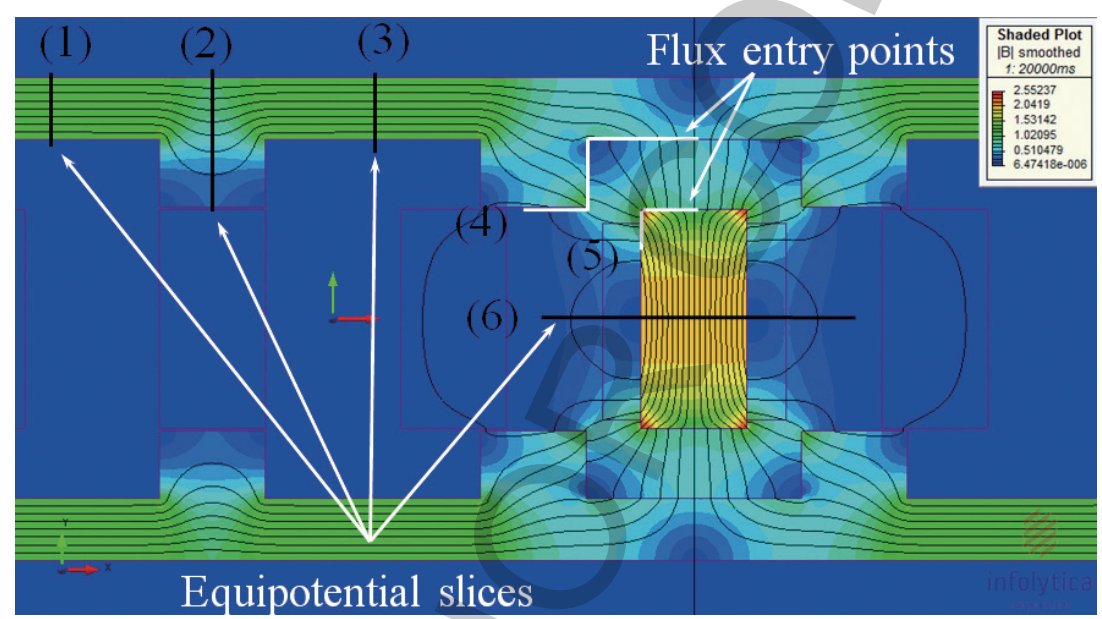

Fig. 2. Magnetic flux plot for a segment of the LSRM for the unaligned position.

of equipotential lines (Fig. 2). By visual inspection of Fig. 2 it is possible to locate equipotential lines on the shaded plot as indicated by the straight lines termed "slices" in reference [7] - these slices will be orthogonal to all the modelled flux paths in the respective parts of the machine. From Fig. 2 it is evident that if an attempt were made to fit smooth and continuous cubic-spline segments between the aforementioned equipotential slices, given only three coordinate points between them, there would be large interpolation errors introduced due to geometric discontinuities and abrupt points of inflection that describe the true flux paths. Therefore it is necessary to subdivide the remaining flux path segments (in between the equipotential lines) further to facilitate accurate cubic-spline interpolation. This is achieved by recognising the very important property of magnetic flux paths that was successfully used in the TAS method [7], namely that each flux path enters magnetic material at a right angle (i.e. normal) to the surface of the material. Once the flux lines are in the material they will follow paths of lowest reluctance. Such magnetic flux path entry points are indicated in Fig. 2.

Figure 3 demonstrates that some of the flux paths can be reasonably accurately approximated by only the three coordinate points, whereas others will require a minimum of four such points. Comparison 


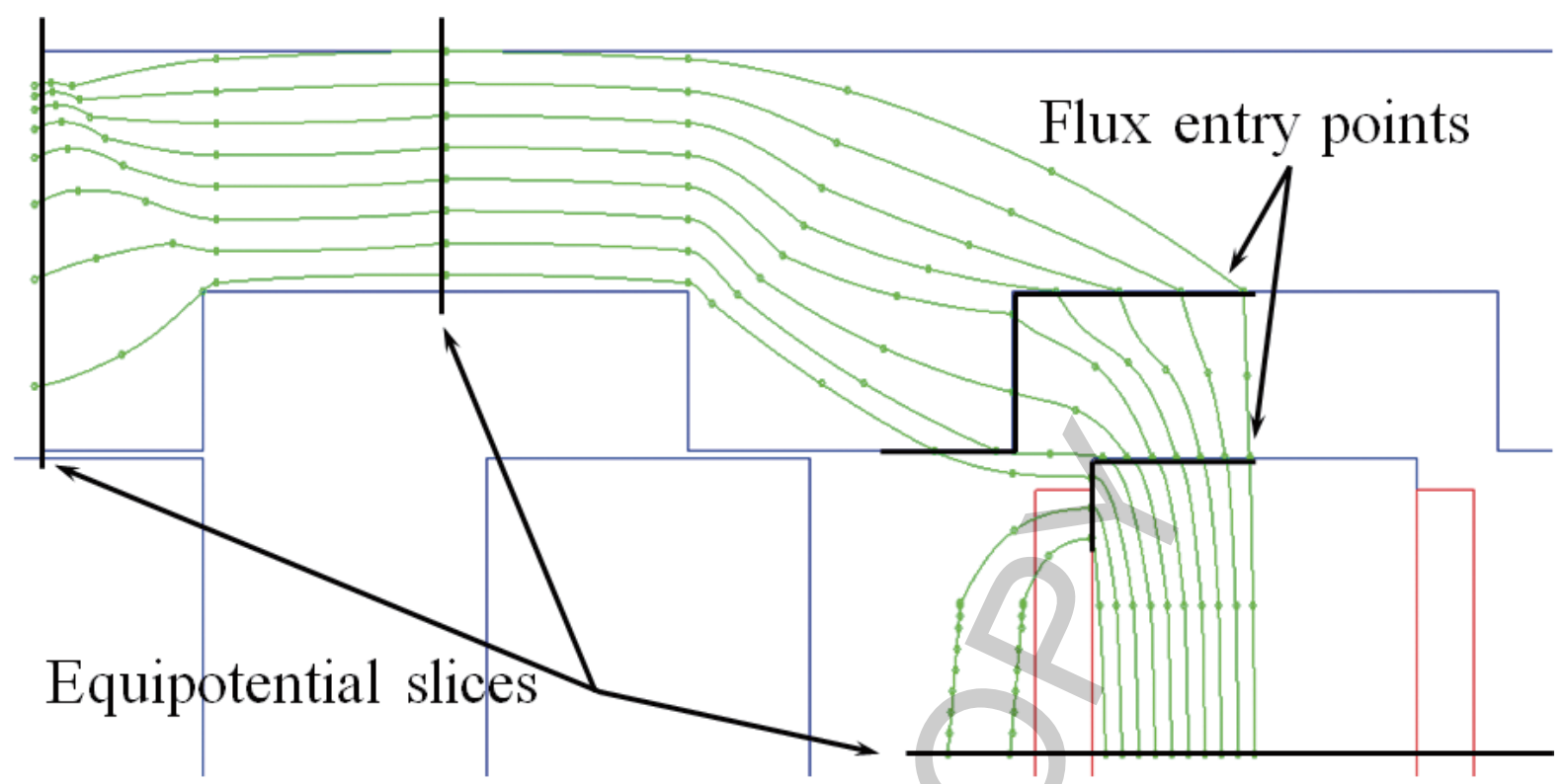

Fig. 3. Cubic-spline based approximation applied to the selected segments of LSRM for the unaligned case.

of Figs 2 and 3 reveals that the segment between slice 2 and slice 3 cannot be accurately interpolated by only three sets of coordinate points. Furthermore, the flux path segment between these two slices contains equipotential magnetic field lines which are not straight lines, but a combination of straight lines as well as circular arcs. To keep the complexity of expressing the flux paths in this particular segment to a minimum, simplified straight line approximations of equipotential magnetic field slices were used (although a more rigorous and accurate method would require construction of other than straight line slices in these regions). This enables simplified mathematical functions of the coordinate points to be defined associated with only major machine dimensions. This simplification might appear to be fairly crude, yet by revisiting the "probable flux path" formulation in reference [3] the justification of this simplification can be made if the final results are in close agreement with more accurate magnetic field analysis techniques such as FEA.

Once the cubic-spline based flux tubes are constructed, and their cord lengths and average crosssectional areas computed, the magneto-motive force ( $\mathrm{mmf}$ ) applied to the excited stator polepiece can be found [1] as

$$
T_{p} \times I=F_{e} \approx 0.5 \times H_{s p} l_{s p}+H_{g} l_{g}+H_{r p} l_{r p}+H_{r y} l_{r y}
$$

where $T_{p}, I, F_{e}, H$ and $l$ are conductor turns per pole, coil current, mmf, magnetic field intensity and cord length of the flux path segment, respectively. The subscripts in Eq. (1) denote segments of the machine: stator pole, air gap, rotor pole and rotor yoke respectively. The relationship is only approximately equal to the applied mmf due to the numerical approximations as described in [1]. For the current study the mmf error was reduced to $1 \%$, or less, of the final iteration before computation was complete. All the geometric quantities for the constructed flux paths will be known and therefore the reluctance values $r_{i}$ for each flux path segment $i$ may be found in the iron parts [1]

$$
r_{i}=\frac{H_{i} l_{i}}{B_{i} A_{i}}
$$




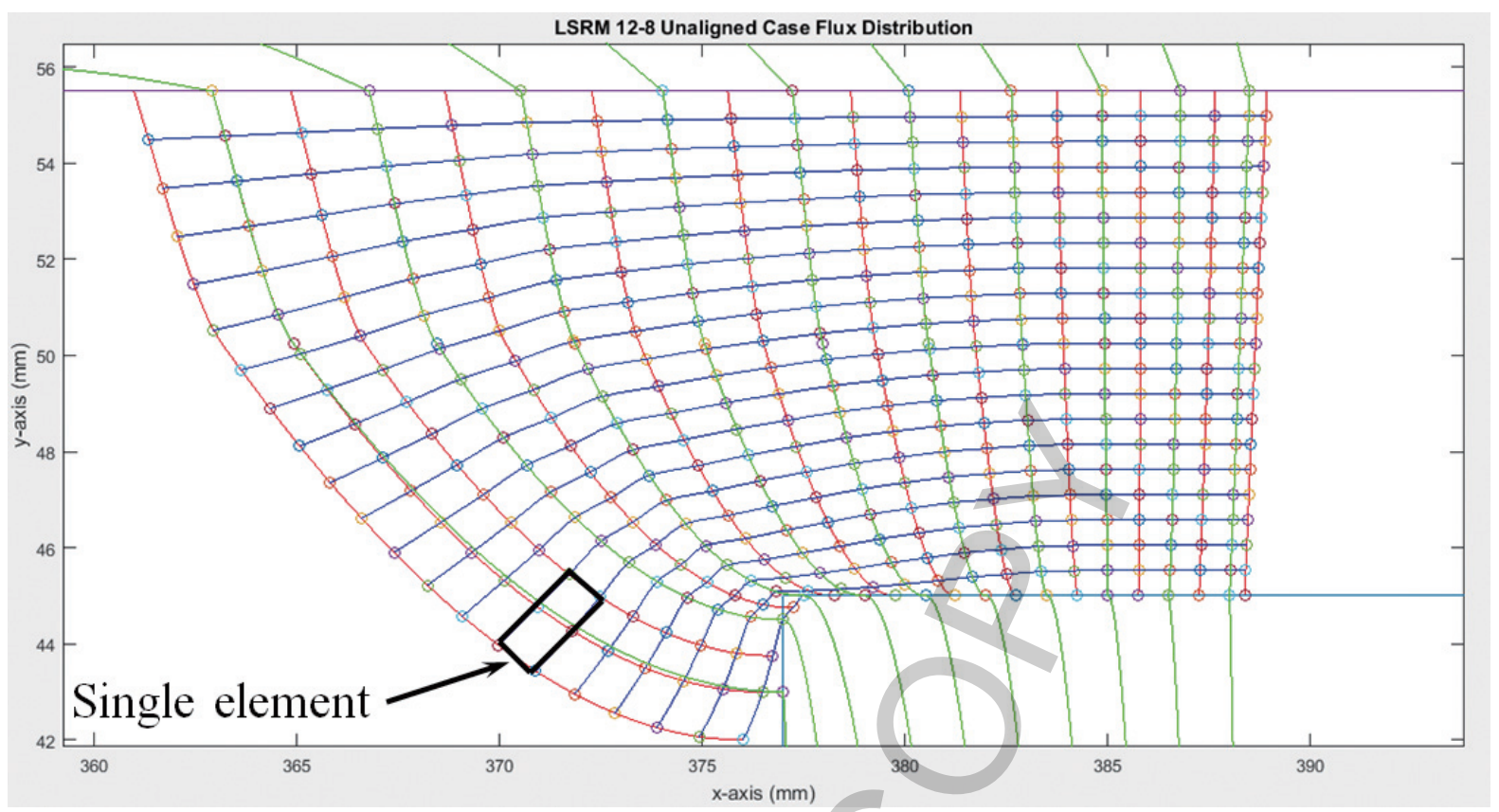

Fig. 4. Approximate subdivision of the LSRM air gap region into tubes of flux and slices of equipotentials.

and in the air gap region

$$
r_{g}=\frac{l_{g}}{\mu_{0} A_{g}}
$$

where $B$ is the magnetic field density and $A$ is average cross-sectional area of the flux path segment. Moreover, because the flux paths are composed of individual flux tube segments connected in series and form complete flux path combined in parallel, the total reluctance $R$ of the complete flux path will be

$$
\frac{1}{R}=\sum \frac{1}{\sum \frac{1}{r_{i}}+\sum \frac{1}{r_{g}}}
$$

Having computed the total reluctance of the flux paths, the flux-linkage function, denoted by $\lambda$, can be found [1]

$$
\lambda=\frac{T_{p} F_{e}}{R}
$$

The important air gap region, for the unaligned translator position, can be further refined to include a larger number of slices (Fig. 4) to provide dual bounds estimate of the air gap reluctance as in the standard TAS approach [7].

In order to get the exact upper and lower bound values of the reluctance of the air gap this region must be subdivided in such a way so that the slices of equipotential lines are orthogonal to the tubes of flux they cut across. As the number of flux tubes and equipotential slices tends towards infinity the orthogonality condition can be approached. However, in practice it is not necessary to approach such a limit as the solution accuracy will converge asymptotically towards the exact values [7] as the number of 


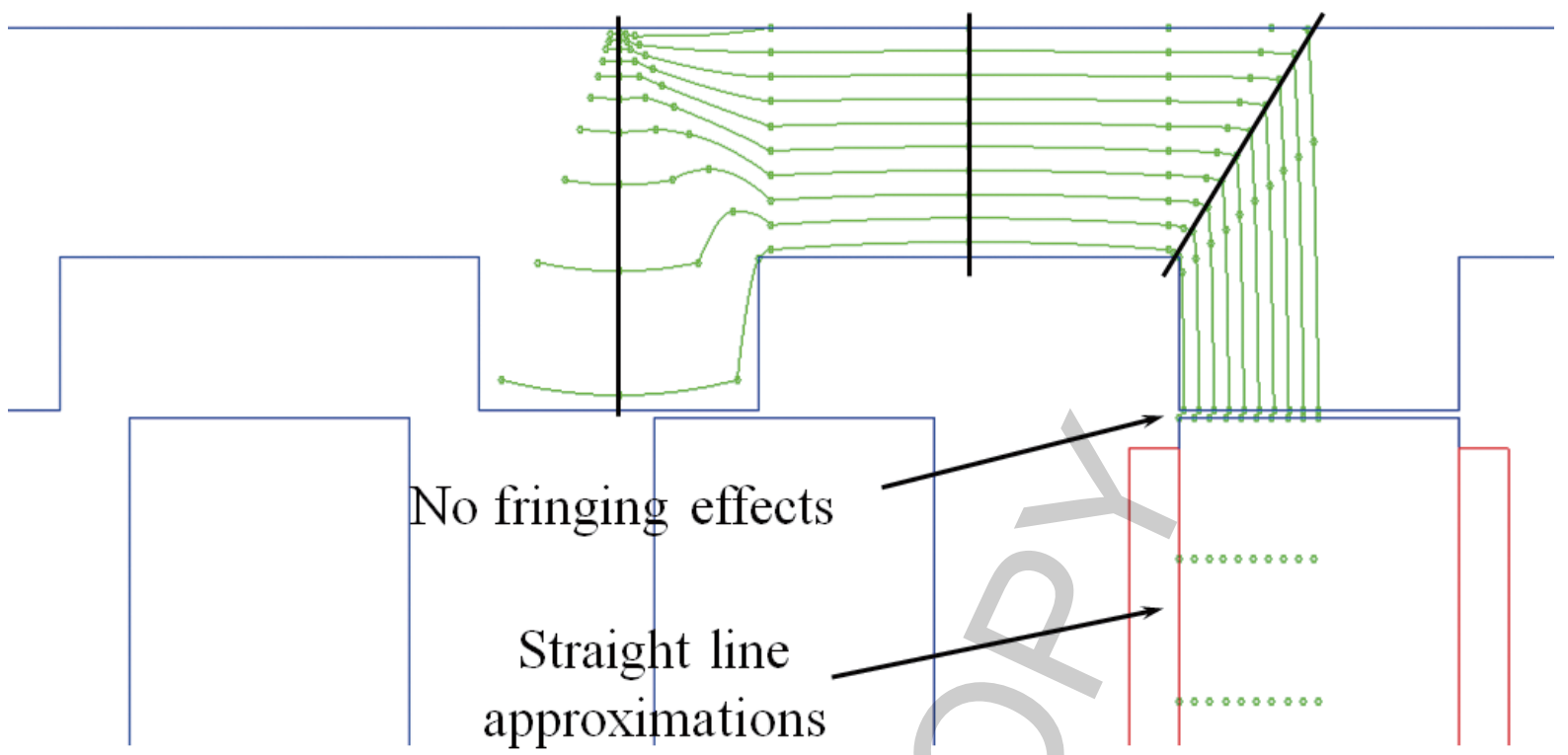

Fig. 5. Aligned translator position probable flux tubes distribution for the LSRM.

tubes and slices grows larger. The demonstrated subdivision level of the air gap region, for the unaligned translator position, into tubes of flux and slices of magnetic field equipotentials approximates this region into a system of curvilinear squares. This subdivision enables determination of upper and lower bound values of reluctance of the air gap region thus increasing confidence in the solution of the flux-linkage function with respect to excitation current.

To compute the total reluctance $R_{t}$ of the flux tubes in the air region, a number of which is denoted by $m$, connected in parallel, the following formula is used [7]

$$
R_{t}=\left[\sum_{i=1}^{m}\left(\sum_{j=1}^{n} \frac{l_{j}}{\mu_{0} A_{i}}\right)^{-1}\right]^{-1}
$$

where each block has length $l_{j}$ and area $A_{i}$, and the formula will give the upper bound value for the reluctance. To compute the total reluctance $R_{s}$ of the flux slices in the air region, a number of which is denoted by $n$, connected in series, the following formula is used [7]

$$
R_{s}=\sum_{j=1}^{n}\left(\sum_{i=1}^{m} \frac{\mu_{0} A_{i}}{l_{j}}\right)^{-1}
$$

and this formula gives the lower bound of the reluctance of the region.

The aligned translator case of the LSRM is shown in Fig. 5 with the probable flux tubes distribution based on cubic-spline approximations as described earlier. It is assumed that flux tubes within the excited stator pole are straight vertical lines for all levels of current, although it is known from the FEA that this is only an approximation as the constant flux lines assume shapes other than straight lines under higher levels of excitation current. It is further assumed that the air gap region, for the aligned translator case, is ideal with no fringing effects. Again, this is only an approximation since the FEA shows some fringing and leakage flux under increased levels of excitation current. All such approximations of assumed flux paths will introduce some error in the final result of the flux-linkage function, yet the complete fluxlinkage map will be - to a large extent - insensitive to these errors as described in reference [9]. 


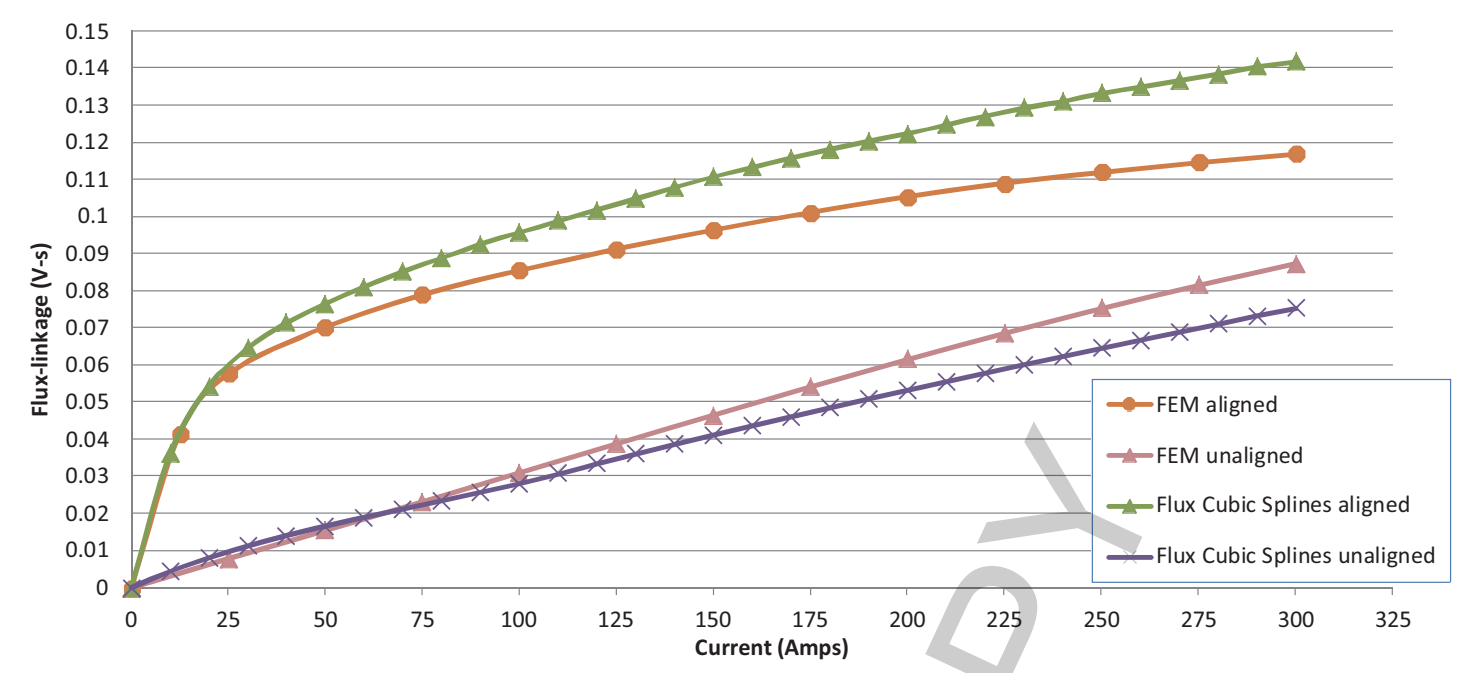

Fig. 6. Flux linkage functions for the aligned and unaligned positions using flux cubic-splines and FEA.

\section{Results}

The improved flux-tube modelling technique has been implemented as an algorithm for performance analysis of LSRM to estimate flux-linkage functions with respect to excitation current (Fig. 6), taking translator position as a parameter. The estimated flux-linkage for the unaligned position is in good agreement with FEA, whereas for the aligned case further model refinement is necessary for accurate determination of the function at higher levels of excitation current. The main contributing factor to the over-estimation of the flux-linkage function for the aligned translator case is that the machine is modelled without taking leakage flux or fringing flux effects into account, as in Fig. 5. In other words, the machine was assumed to have an ideal flux-tube distribution pattern for the whole range of coil currents. It can be seen that this assumption consistently over predicts the flux-linkage function for the aligned translator case of the analysed machine.

The unaligned translator position flux-linkage function of the improved flux-tube method is representative of general flux-linkage function for this translator position in that it is a near-linear function with respect to excitation current. It is certainly possible to saturate machine iron parts at the unaligned position so that the unaligned case flux-linkage function becomes non-linear; for this to happen, however, the current levels would be too high for any practical purposes. The unaligned case flux-linkage function, shown in Fig. 6, is $14 \%$ below the curve obtained from FEA at the current level of 300 amperes, which is 1.2 times higher than the specified design current of the machine. This result is of similar accuracy compared with the alternative flux-tube modelling approaches described in references [1-5].

The TAS refinement of the new flux-tube method was used to obtain the dual bounds approximations of the reluctance values of the air-gap region for the unaligned translator position of the LSRM. Figure 7 shows the flux-linkage function obtained from this analysis.

As was stated above, the dual bound approximations of the unaligned case flux-linkage function provide useful results which can be used to select a more conservative design from the two alternative answers. Alternatively, the two results can be combined to give an averaged value with upper and lower limits serving as a guarantee that this average value is in between the limits.

The improved flux-tube method was tested for consistency of resulting flux-linkage functions when geometry parameters of the analysed LSRM were varied. It is known, from [10], that by increasing the 


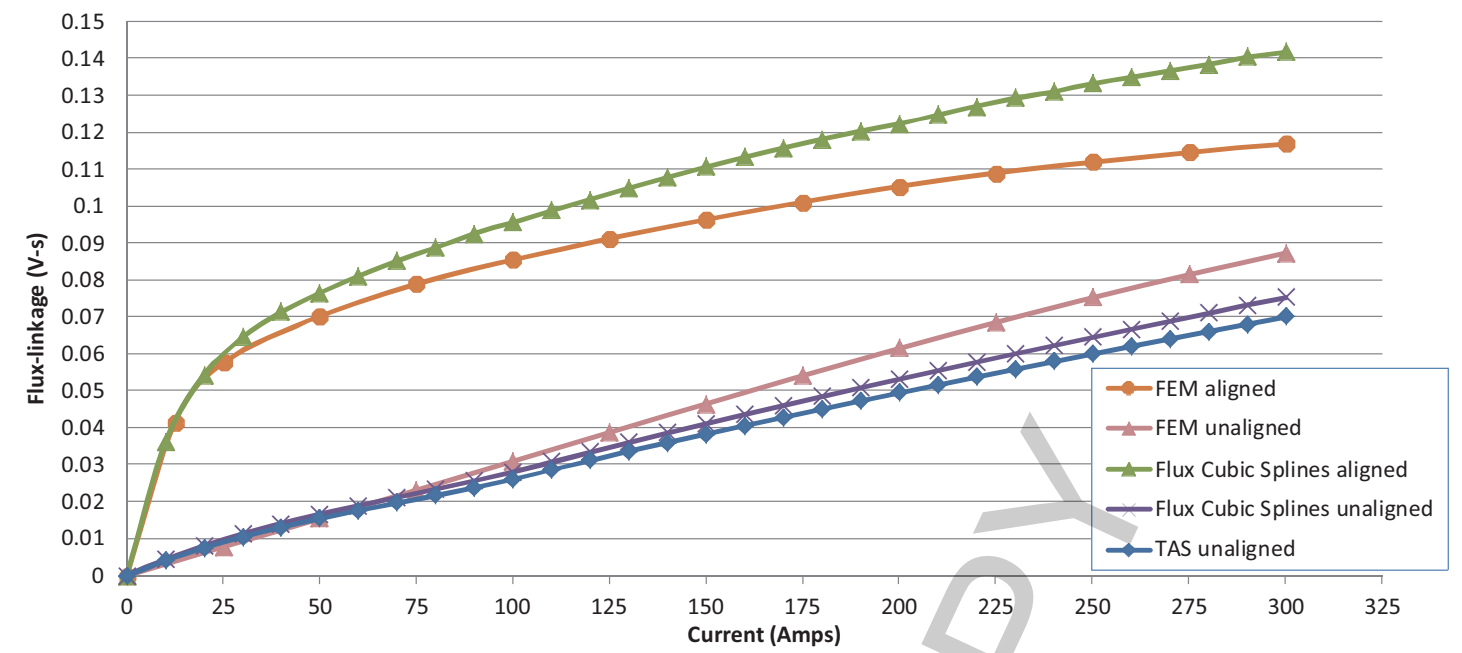

Fig. 7. Flux-linkage functions for the aligned and unaligned translator instances including the unaligned instance obtained with TAS refinement.

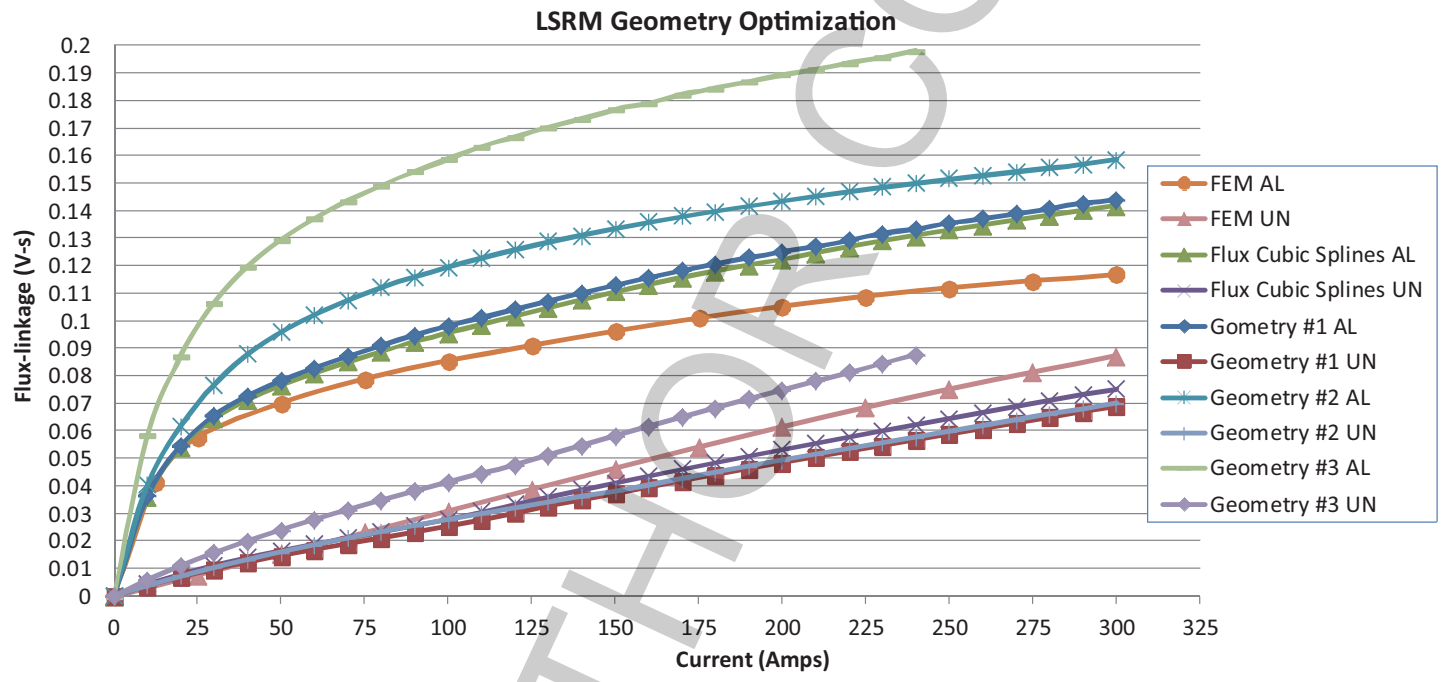

Fig. 8. Flux linkages for the aligned and unaligned positions using flux cubic-splines and FEA.

translator pole height, in other words the air-gap length for the unaligned translator position, it is possible to reduce the unaligned flux-linkage level and thus increase the surface area of the entire flux-linkage map. This has a positive impact on the machine performance as it results in higher tractive force output compared to a machine which has a smaller air-gap region, as shown in Fig. 8, for the unaligned case of machine geometry \#1 where stator pole height was increased by $15 \%$.

By following the switched reluctance machine design guidelines in [11], further improvements in the LSRM performance are possible. For example, by increasing the back-iron thickness of the translator parts of the machine by $20 \%$, the aligned translator position flux-linkage function is increased markedly for a given ampere-turns parameter value of the machine. This effect is shown for the analysed machine geometry \#2 aligned translator case (Fig. 8). Finally, the new flux-tube method can be used to optimize 
the conductor-turns parameter of the LSRM. For illustration purposes the initial 48 conductor-turns coil of the machine was changed to 60 conductor-turns coil with the ampere-turns parameter being held constant. The flux-tube method indicates that the machine flux-linkage function is increased substantially as shown for the geometry \#3 aligned translator case (Fig. 8). This result was to be expected since the flux linkage is a function which increases in proportion to the number of conductors linked with the flux paths [1]; therefore, by increasing the number of turns, the surface area of the complete threedimensional flux-linkage map is enlarged. This enlargement results in a considerable increase in the tractive force of the machine, but reduced excitation current levels compared to the original conductorturns value.

\section{Discussion and conclusions}

From the methodology and results sections of the paper the following conclusions with regard to the improved flux-tube modelling method can be drawn.

1. The improved flux-tube modelling approach reduces the ambiguities in the construction of fluxtubes in electromechanical devices, compared to other such techniques. The uncertainty regarding the selection of machine dimensions that will define the coordinate points through which each flux path should pass also clarified. The proposed approach also reduces the required number of geometric parameters of the modelled electromechanical device necessary to establish an accurate mathematical expression for the flux-tubes distribution, compared to the equivalent field modelling methods.

2. Accurate determination of the flux-tube cord lengths is possible with the improved flux-tube method since all the information needed to achieve this is available in the form of cubic splines. Computationally efficient numerical integration is then performed to find the cord lengths. Therefore an exact cord length value can be found for all assumed flux-tubes compared to average cord length values used in the equivalent field modelling methods.

3. A complete flux-linkage map, including the magnetic saturation effects, can be constructed for an SR machine using the new flux-tube method. The results obtained from the method are in good agreement with the finite element analysis. However, the new method does not account for leakage flux or fringing flux effects, around the excited phase windings, occurring at high levels of excitation current. Therefore further improvements to the proposed flux-tube modelling approach will be beneficial in providing better accuracy of flux-linkage representation at high levels of excitation current when the leakage flux becomes considerable. This leakage flux reduces the available useful output power levels of a given machine and should be taken into account for more accurate machine characterisation.

4. Incorporating the proposed flux-tube method into design optimization algorithms is possible and advantageous since the method is computationally efficient, with a complete three-dimensional flux-linkage map construction taking 50 seconds to compute. A comparable FEA was found to require 250 seconds to achieve the same flux-linkage map, however with inherently much higher accuracy. This computational advantage of the proposed flux-tube method can be exploited during initial optimization and search stages when a large design space must be explored as in [12]. The method is especially useful when new magnetic circuit topologies of novel SR machines are being analysed as in [13]. During this stage of design search and optimization the computational efficiency of the improved flux-tube method can outweigh the approximate nature of the results obtained. 


\section{References}

[1] R. Krishnan, Switched Reluctance Motor Drives: Modelling, Simulation, Analysis, Design, and Applications, CRC Press LLC, Boca Raton Florida, 2001.

[2] J.M. Kokernak and D.A. Torrey, Magnetic Circuit Model for the Mutually Coupled Switched-Reluctance Machine, IEEE Transactions on Magnetics 36(2) (2000).

[3] H.C. Roters, Electromagnetic Devices, $1^{\text {st }}$ Edition, 1941, John Wiley \& Sons Inc.

[4] J. Corda and J.M. Stephenson, Analytical Estimation of the Minimum and Maximum Inductances of a Double Salient Motor, International Conference on Stepping Motors and Systems, The Department of Electrical and Electronic Engineering, University of Leeds, 1979.

[5] U.S. Deshpande, J.J. Cathey and E. Richter, High-Force Density Linear Switched Reluctance Machine, IEEE Transactions on Industry Applications 31(2) (1995).

[6] J.K. Sykulski, Dual Field Modelling Using Tubes and Slices, Advances in Engineering Software, Vol. 21, pp. 21-26, Elsevier Science Limited, 1995.

[7] P. Hammond and J.K. Sykulski, Engineering Electromagnetism: Physical Processes and Computation, Oxford University Press, 1994

[8] T.J.E. Miller, Nonlinear theory of the switched reluctance motor for rapid computer-aided design, IEE Proceedings 137(6) (1990).

[9] J.M. Stephenson and J. Corda, Computation of torque and current in doubly salient reluctance motors from nonlinear magnetisation data, in Proceedings IEE, Vol. 126, No. 5, May 1979.

[10] P.J. Lawrenson, J.M. Stephenson, P.T. Blenkinsop, J. Corda and N.N. Fulton, Variable-speed switched reluctance motors, in IEE Proceedings, Vol. 127, Pt. B, No. 4, July 1980.

[11] T.J.E. Miller, Switched Reluctance Motors and Their Control, ISBN: 0198593872, Oxford University Press, Oxford, UK.

[12] J. Choi, J. Lee and Y. Kim, Geometric design of pole arcs considering electric parameters in switched reluctance motor, International Journal of Applied Electromagnetics and Mechanics 19(1-4) (2004), 275-279.

[13] E. El-Kharashi, Improving the energy conversion process in the switched reluctance motors, International Journal of Applied Electromagnetics and Mechanics 41(4) (2013), 375-387, DOI: 10.3233/JAE-121619. 